\title{
Identification of novel small molecule inhibitors against nsP2 protease of CHIKV through a molecular modeling approach
}

Sutanu Mukhopadhyay ( $\nabla$ sutanubermensch@gmail.com )

Ramakrishna Mission Vivekananda Centenary College, Rahara, West Bengal, India https://orcid.org/0000-0001-8243-2138

\section{Research Article}

Keywords: CHIKV, nsP2 protease, de novo design, docking, artificial neural network, ADMET

Posted Date: November 9th, 2021

DOI: https://doi.org/10.21203/rs.3.rs-1056189/v1

License: (c) (i) This work is licensed under a Creative Commons Attribution 4.0 International License. Read Full License 


\section{Abstract}

Chikungunya is a tropical viral disease spread by the female Aedes mosquitoes infected with the Chikungunya virus (CHIKV). Nonstructural protein 2 (nsp2) plays a crucial role in the viral life cycle by its proteolytic activity and hence it is one of the most important drug targets. There is currently no permanent treatment available to tackle the infection. In this molecular modeling-based study, a combination of de novo ligand design, molecular docking, and ADMET-based screening is employed to identify novel inhibitor molecules targeting the active site of nsP2 protease of the CHIKV. A set of molecules have been shortlisted as potential inhibitors based on their binding affinity and drug-likeness score. Further experimental validation is required to verify the potency of the proposed leads against CHIKV nsp2 protease activity to combat the infection.

\section{Introduction}

Chikungunya is a viral infectious disease spread by the bites of infected female Aedes mosquitos. It produces fever, joint aches and swelling, muscle pain, muscle pain, nausea, headache, and rash. It was initially reported in Southern Tanzania, and it has now been detected in over forty countries worldwide (1-3). It is an RNA virus, belongs to the Togaviridae family's alphavirus genus. The disease is mostly transmitted by two mosquito species known as Aedes aegypti and Aedes albopictus (4). The word "chikungunya" is originated from a phrase in the Makonde language that means "that which bends up," and refers to the hunched posture of people suffering from joint discomfort (arthralgia) (1).

The virus's genome is translated into nine proteins, five of which are structural (one capsid protein (C), two envelope glycoproteins (E1, E2), and two peptides, E3 and 6K) and four of which are non-structural (nsP1, nsP2, nsP3, and nsP4) $(5,6)$. Among the structural proteins, capsid protein initiates the viral life cycle within the host cell via autoproteolysis, whilst envelope glycoproteins aid in the viral entrance (7). nsP1 and nsP3 synthesize the negative sense of the RNA strand, while nsP4 controls virion polymerization in the host cell. The multifunctional protein $\mathrm{nsP} 2$ is a member of the papain superfamily of cysteine proteases, which is well recognized for its proteolytic activity and involvement in viral replication. Therefore, $\mathrm{nsP} 2$ is one of the most promising drug targets for developing drugs against the infection (8). Several computational and experimental studies have been conducted in search of inhibitors of nsP2 through different routes such as molecular modeling, structure-based drug design, molecular docking, and MD simulation, pharmacophore mapping, biological evaluation, and many more $(9-17)$.

Deep learning contributes to the field of drug discovery in multiple ways from searching potential binding pockets on the surface of the protein (18-20) to generating novel molecules based on the protein pocket information (21-24). LiGANN uses deep learning-based generative adversarial networks (GANs) to generate ligands taking the protein structure information (21).

In this in silico study, the crystal structure of CHIKV nsP2 protease was obtained from protein databank (25) and, based on the active site information, novel lead compounds were created using LiGANN, a de novo drug design tool based on generative neural network (GNN) (21). All of the suggested molecules are then manually docked to the nsP2 active site using PyRx, an autodock-embedded software $(26,27)$. The highest scoring ligands' ADMET characteristics were evaluated using the SwissADME webserver (28). Further studies are needed to investigate the efficiency of the suggested compounds in treating chikungunya virus infection.

\section{Materials And Methods}

Protein structure retrieval: The crystal structure of the chikungunya virus nsP2 protease has been downloaded from the RCSB PDB website (PDB ID: 3TRK). The crystallographic water molecules and the ions are removed and the protein surface is visualized using PyMol (29).

de novo ligand design: The prepared protein pdb file has been uploaded as input in the LiGANN webserver (https://playmolecule.com/LiGANN/), number of ligand shape generations and decoding per shape has been set as 10 and 10 respectively. A grid box has been generated centering the catalytic dyad (CYS 1013 and HIS 1083) of the protein.

Molecular docking: Then the protein has been processed and converted into. pdbqt using PyRx "Make macromolecule" utility. A total of 88 molecules have been generated by LiGANN, using Open Babel, the SMILES ID of the compounds are converted into .sdf file format (30). All the downloaded compounds have been energy minimized and converted into. pdbqt format before docking using PyRx. A grid box has been created around the catalytic dyad (CYS 1013 and HIS 1083) and docking has been performed using the vina wizard utility of PyRx. The 2D depiction of interactions of the protein-ligand complexes has been created using Discovery Studio visualizer (31). 
ADMET prediction: ADMET stands for Absorption, Digestion, Metabolism, Excretion, and Toxicity, this is a measure of drug metabolism and pharmacokinetics (DMPK) which is crucial for drug discovery. ADMET and physicochemical properties, drug-likeness, synthetic accessibility of the top-scoring leads have been estimated using the SwissADME web server.

\section{Results And Discussions}

Protein structure retrieval: The crystal structure of the chikungunya virus nsP2 protease (3TRK) is downloaded in .pdb file format consists of 324 amino acids and has a resolution of $2.40 \AA$. The three-dimensional structure is visualized in cartoon representation, the catalytic dyads are shown in licorice representation (Figure 1).

de novo ligand design: The modified pdb file is given as input on the LiGANN server, which results in 88 molecules based on the protein active site information. The molecules belong to different chemical classes so that there's a diverse group of ligands.

Molecular docking: Among the 88 ligands, 17 ligands have a binding affinity score of $\leq-6.0 \mathrm{Kcal} / \mathrm{mol}$; but all of them are not interacting with at least one of the catalytic dyad residues. In the case of the best scoring lead (Figure 2.A), molecule $A(-7.6 \mathrm{kcal} / \mathrm{mol})$ has a total of seven non-bonding interactions with the neighboring amino acids at the active site of the protein including two pi-sulfur interactions with CYS 1013(distance: $4.91 \AA$ ) and MSE 1238 (Selenomethionine), a hydrogen bond with TYR 1047, two pi-sigma interactions with ALA 1046 and MSE 1242, two pi-alkyl interactions with ALA 1046 and TYR 1079. Molecule $D, H, L, M, N$ interacts with CYS 1013 with pi-sulfur nonbonded interactions while molecule $E$ interacts through pi-alkyl interaction. A list of all the interactions is listed in Table 1 and all the 2D protein-ligand interaction images are shown in Figure 2.

ADMET prediction: The SwissADME web-server was used to determine various physicochemical parameters, leadlikeness, and synthetic accessibility of the top 17 lead compounds, as shown in Table 2. All the molecules have a molecular weight of $\leq 500 \mathrm{~g} / \mathrm{mol}$, the number of hydrogen acceptor atoms (HA) and hydrogen donor atoms (HD) are $\leq 10$ and $\leq 5$ respectively which are the criteria in Lipinski's rule of five for drug-likeness. According to Veber's rule of drug-likeness, a drug-like molecule should have $\leq 10$ rotatable bonds and Topological Polar Surface Area (TPSA) of $\leq 140 \AA^{2}$, most of the molecules passed the first criterion (Molecule $A, B, D, E, F, G, H, I, L, N, O$ ) and all the molecules satisfied the second criterion. Log Po/w is a measure of lipophilicity of the molecules, here a consensus value is given as an average of five values predicted in different manners. A drug-like molecule must have optimal aqueous solubility; most compounds are moderately soluble in water. The bioavailability score indicates the percentage of the drug that may reach the target location; all molecules have a bioavailability score of 0.55 . After summing up all the information related to leadlikeness, 6 molecules are found as leadlike (Molecule A, B, D, F, G, N) according to SwissADME predictions. Synthetic accessibility score, predicted by the server signifies the ease of synthesizing the molecules in the laboratory, where score 1 means the molecule can be synthesized very easily and 10 means it is very difficult to synthesize the molecule.

\section{Conclusions}

Chikungunya is a threat to public health worldwide. This study identifies several novel leads which are effective against the nsP2 viral protein of the virus. Further experimental studies should be conducted to verify their potency and discover effective drugs against the disease.

\section{Declarations}

\section{Competing interests:}

There are no conflicts of interest related with this publication, and no financial assistance has been provided for this work.

\section{References}

1. Chikungunya fact sheet [Internet]. [cited 2021 Nov 6]. Available from: https://www.who.int/news-room/factsheets/detail/chikungunya

2. Robinson MC. An epidemic of virus disease in southern province, tanganyika territory, in 1952-1953. i. clinical features. Trans R Soc Trop Med Hyg. 1955;49(1):28-32.

3. Bodenmann P, Genton B. Chikungunya: an epidemic in real time. Lancet. 2006 Jul 15;368(9531):258. 
4. Sourisseau M, Schilte C, Casartelli N, Trouillet C, Guivel-Benhassine F, Rudnicka D, et al. Characterization of Reemerging Chikungunya Virus. PLOS Pathog [Internet]. 2007 Jun [cited 2021 Nov 6];3(6):e89. Available from:

https://journals.plos.org/plospathogens/article?id=10.1371/journal.ppat.0030089

5. Strauss EG, Strauss JH. Structure and Replication of the Alphavirus Genome. The Togaviridae and Flaviviridae. 1986;35-90.

6. Takkinen K. Complete nucleotide sequence of the nonstructural protein genes of Semliki Forest virus. Nucleic Acids Res [Internet]. 1986 Jul 11 [cited 2021 Nov 6];14(14):5667. Available from: /pmc/articles/PMC311584/?report=abstract

7. Simizu B, Yamamoto K, Hashimoto K, Ogata T. Structural proteins of Chikungunya virus. J Virol [Internet]. 1984 Jul [cited 2021 Nov 6];51(1):254-8. Available from: https://journals.asm.org/doi/abs/10.1128/jvi.51.1.254-258.1984

8. Sreejith R, Rana J, Dudha N, Kumar K, Gabrani R, Sharma SK, et al. Mapping interactions of Chikungunya virus nonstructural proteins. Virus Res. 2012 Oct 1;169(1):231-6.

9. Singh KD, Kirubakaran P, Nagarajan S, Sakkiah S, Muthusamy K, Velmurgan D, et al. Homology modeling, molecular dynamics, epharmacophore mapping and docking study of Chikungunya virus nsP2 protease. J Mol Model 2011181 [Internet]. 2011 Mar 29 [cited 2021 Nov 6];18(1):39-51. Available from: https://link.springer.com/article/10.1007/s00894-011-1018-3

10. Fros JJ, van der Maten E, Vlak JM, Pijlman GP. The C-Terminal Domain of Chikungunya Virus nsP2 Independently Governs Viral RNA Replication, Cytopathicity, and Inhibition of Interferon Signaling. J Virol [Internet]. 2013 Sep 15 [cited 2021 Nov 6];87(18):10394-400. Available from: https://journals.asm.org/doi/abs/10.1128/JVI.00884-13

11. Lucas-Hourani M, Lupan A, Desprès P, Thoret S, Pamlard O, Dubois J, et al. A phenotypic assay to identify chikungunya virus inhibitors targeting the nonstructural protein nsP2. J Biomol Screen [Internet]. 2013 Feb 14 [cited 2021 Nov 6];18(2):172-9. Available from: https://journals.sagepub.com/doi/full/10.1177/1087057112460091

12. Nguyen PTV, Yu H, Keller PA. Identification of chikungunya virus nsP2 protease inhibitors using structure-base approaches. J Mol Graph Model. 2015 Apr 1;57:1-8.

13. Kumar P, Kumar D, Giri R. Targeting the nsp2 Cysteine Protease of Chikungunya Virus Using FDA Approved Library and Selected Cysteine Protease Inhibitors. Pathog 2019, Vol 8, Page 128 [Internet]. 2019 Aug 15 [cited 2021 Nov 6];8(3):128. Available from: https://www.mdpi.com/2076-0817/8/3/128/htm

14. Khan N, Bhat R, Patel AK, Ray P. Discovery of small molecule inhibitors of chikungunya virus proteins (nsP2 and E1) using in silico approaches. https://doi.org/101080/0739110220201731602 [Internet]. 2020 [cited 2021 Nov 6];39(4):1373-85. Available from: https://www.tandfonline.com/doi/abs/10.1080/07391102.2020.1731602

15. Ivanova L, Rausalu K, Žusinaite E, Tammiku-Taul J, Merits A, Karelson M. 1,3-Thiazolbenzamide Derivatives as Chikungunya Virus nsP2 Protease Inhibitors. ACS Omega [Internet]. 2021 Mar 2 [cited 2021 Nov 6];6(8):5786-94. Available from:

https://pubs.acs.org/doi/abs/10.1021/acsomega.0c06191

16. Ivanova L, Rausalu K, Ošeka M, Kananovich DG, Žusinaite E, Tammiku-Taul J, et al. Novel Analogues of the Chikungunya Virus Protease Inhibitor: Molecular Design, Synthesis, and Biological Evaluation. ACS Omega [Internet]. 2021 Apr 27 [cited 2021 Nov 6];6(16):10884-96. Available from: https://pubs.acs.org/doi/abs/10.1021/acsomega.1c00625

17. Islamuddin M, Afzal O, Khan WH, Hisamuddin M, Altamimi ASA, Husain I, et al. Inhibition of Chikungunya Virus Infection by 4Hydroxy-1-Methyl-3-(3-morpholinopropanoyl)quinoline-2(1 H)-one (QVIR) Targeting nsP2 and E2 Proteins. ACS Omega [Internet]. 2021 Apr 13 [cited 2021 Nov 6];6(14):9791-803. Available from: https://pubs.acs.org/doi/abs/10.1021/acsomega.1c00447

18. Jiménez J, Doerr S, Martínez-Rosell G, Rose AS, De Fabritiis G. DeepSite: protein-binding site predictor using 3D-convolutional neural networks. Bioinformatics [Internet]. 2017 Oct 1 [cited 2021 Nov 6];33(19):3036-42. Available from:

https://academic.oup.com/bioinformatics/article/33/19/3036/3859178

19. Simonovsky M, Meyers J. Deeply Tough: Learning Structural Comparison of Protein Binding Sites. J Chem Inf Model [Internet]. 2020 Apr 27 [cited 2021 Nov 6];60(4):2356-66. Available from: https://pubs.acs.org/doi/full/10.1021/acs.jcim.9b00554 
20. Aggarwal R, Gupta A, Chelur V, Jawahar C V., Priyakumar UD. DeepPocket: Ligand Binding Site Detection and Segmentation using 3D Convolutional Neural Networks. J Chem Inf Model [Internet]. 2021 [cited 2021 Nov 6]; Available from:

https://pubs.acs.org/doi/abs/10.1021/acs.jcim.1c00799

21. Skalic M, Sabbadin D, Sattarov B, Sciabola S, De Fabritiis G. From Target to Drug: Generative Modeling for the Multimodal StructureBased Ligand Design. Mol Pharm. 2019 Oct 7;16(10):4282-91.

22. Olivecrona M, Blaschke T, Engkvist O, Chen H. Molecular de-novo design through deep reinforcement learning. J Cheminform [Internet]. 2017 Sep 4 [cited 2021 Nov 6];9(1):1-14. Available from: https://jcheminf.biomedcentral.com/articles/10.1186/s13321-0170235-x

23. Segler MHS, Kogej T, Tyrchan C, Waller MP. Generating focused molecule libraries for drug discovery with recurrent neural networks. ACS Cent Sci [Internet]. 2018 Jan 24 [cited 2021 Nov 6];4(1):120-31. Available from:

https://pubs.acs.org/doi/abs/10.1021/acscentsci.7b00512

24. Gupta A, Müller AT, Huisman BJH, Fuchs JA, Schneider P, Schneider G. Generative Recurrent Networks for De Novo Drug Design. Mol Inform [Internet]. 2018 Jan 1 [cited 2021 Nov 6];37(1-2):1700111. Available from:

https://onlinelibrary.wiley.com/doi/full/10.1002/minf.201700111

25. Structure of the Chikungunya virus nsP2 protease... - Google Scholar [Internet]. [cited 2021 Nov 6].

26. Dallakyan S, Olson AJ. Small-molecule library screening by docking with PyRx. Methods Mol Biol [Internet]. 2015 [cited 2021 Nov 6];1263:243-50. Available from: https://pubmed.ncbi.nlm.nih.gov/25618350/

27. Cosconati S, Forli S, Perryman AL, Harris R, Goodsell DS, Olson AJ. Virtual Screening with AutoDock: Theory and Practice. Expert Opin Drug Discov [Internet]. 2010 Jun [cited 2021 Nov 6];5(6):597-607. Available from: https://pubmed.ncbi.nlm.nih.gov/21532931/

28. Daina A, Michielin O, Zoete V. SwissADME: a free web tool to evaluate pharmacokinetics, drug-likeness and medicinal chemistry friendliness of small molecules. Sci Rep [Internet]. 2017;7(1):42717. Available from: https://doi.org/10.1038/srep42717

29. Schrödinger, LLC. The \{PyMOL\} Molecular Graphics System, Version 1.8. 2015 Nov.

30. O’Boyle NM, Banck M, James CA, Morley C, Vandermeersch T, Hutchison GR. Open Babel: An Open chemical toolbox. J Cheminform [Internet]. 2011 Oct 7 [cited 2021 Nov 7];3(10):1-14. Available from: https://jcheminf.biomedcentral.com/articles/10.1186/1758-2946-3-33

31. Dassault Systèmes BIOVIA, Discovery Studio Modeling Environment, Release 2020

\section{Tables}


Table 1

Details of the binding affinities and interacting amino acid residues for top 17 ligands

\begin{tabular}{|c|c|c|c|}
\hline Molecule & SMILES & $\begin{array}{l}\text { Binding } \\
\text { affinity } \\
\text { score } \\
\text { (kcal/mol) }\end{array}$ & Neighboring interacting residues \\
\hline A & CN1CCC(CC1)C1ONC(N1)[C@@H]1CNc2c(CC1)cccc2 & -7.6 & $\begin{array}{l}\text { CYS 1013. ALA 1046(2), TYR 1047, TYR } \\
\text { 1079, MSE 1238, MSE } 1242\end{array}$ \\
\hline B & $\mathrm{N} 1 \mathrm{CC}(\mathrm{C} 1) \mathrm{CNC} 1 \mathrm{C}(\mathrm{Cc} 2 \mathrm{ccccc} 2) \mathrm{Cc} 2 \mathrm{n} 1 \mathrm{ncn} 2$ & -7.0 & TYR 1047, TYR 1079, TRP 1084, ASP 1246 \\
\hline C & $\operatorname{CCC}(\mathrm{NC}(=0) \operatorname{CSc} 1 \mathrm{nnc}(\mathrm{s} 1) \operatorname{NCCCN}(\operatorname{Cc} 1 \operatorname{ccccc} 1) \mathrm{C}) \mathrm{C}$ & -6.7 & $\begin{array}{l}\text { TRP 1014, ALA 1046, TYR 1047 (3), TYR } \\
1079 \text { (2), ASN 1082, TRP 1084, MSE } 1242\end{array}$ \\
\hline D & Sc1nnc(n1CCC1CNCC1)CCc1 ccccc1 & -6.7 & $\begin{array}{l}\text { CYS 1013, ALA 1046, LEU 1205, MSE } 1242 \text {, } \\
\text { ASP } 1246\end{array}$ \\
\hline $\mathrm{E}$ & $\mathrm{CNCCCC}(=0) \mathrm{N} 1 \mathrm{CCN}(\mathrm{c} 2 \mathrm{nnc}(\mathrm{CCCN} 3 \mathrm{CCCC} 3) \mathrm{n} 2 \mathrm{C}) \mathrm{CC} 1$ & -6.7 & $\begin{array}{l}\text { CYS 1013, ALA 1046, TYR 1047, GLU 1050, } \\
\text { TYR 1079, ASN 1082, LYS 1091 } \\
\text { (unfavorable), MSE } 1242\end{array}$ \\
\hline $\mathrm{F}$ & $\mathrm{CN}(\mathrm{C}) \mathrm{CC}(\mathrm{CNc} 1 \mathrm{cccnn} 1) \mathrm{C} 1 \mathrm{CCC} 2 \mathrm{ccccc} 21$ & -6.6 & $\begin{array}{l}\text { ALA 1046, TYR } 1047 \text { (2), SER 1048, TYR } \\
1079\end{array}$ \\
\hline G & $\mathrm{CC} 1 \mathrm{CC}(\mathrm{CN}) \mathrm{CN} 1 \mathrm{c} 1 \mathrm{nnc}(\mathrm{Cc} 2 \mathrm{ccccc} 2) \mathrm{n} 1 \mathrm{CC} 1 \mathrm{CC} 1$ & -6.5 & ASN 1082, TRP 1084, LEU 1205 \\
\hline $\mathrm{H}$ & $\operatorname{COCC}(\mathrm{C}) \mathrm{Nc} 1 \mathrm{cc} 2 \operatorname{ccccc} 2 \mathrm{nn} 1$ & -6.4 & $\begin{array}{l}\text { ASN } 1011 \text { (2), CYS 1013, ALA } 1046 \text { (2), } \\
\text { TYR 1047, TYR 1079, ASN 1082, MSE } 1242\end{array}$ \\
\hline I & $\mathrm{CN}(\mathrm{C}) \operatorname{CCN}(\mathrm{CCc} 1 \mathrm{ccccc} 1) \operatorname{Cc} 1 \mathrm{ccc} n 1$ & -6.3 & $\begin{array}{l}\text { TYR 1047, TYR } 1079 \text { (2), TRP 1084, LEU } \\
\text { 1205, ASP } 1246\end{array}$ \\
\hline J & $\operatorname{CNCCCNC}(=0) \operatorname{CCC}(\mathrm{CN}(\mathrm{C}) \mathrm{C}) \mathrm{NC}(=0) 0 \mathrm{Cc} 1 \mathrm{ccccc} 1$ & -6.3 & $\begin{array}{l}\text { TYR 1047, TYR 1079, ASN 1082, TRP } 1084 \\
\text { LYS } 1091\end{array}$ \\
\hline $\mathrm{K}$ & $\mathrm{CC}(\mathrm{C}) \mathrm{NC}(=0) \mathrm{NCCCC}(=0) \mathrm{NCCN}(\mathrm{C}) \mathrm{Cc} 1 \mathrm{ccco} 1$ & -6.2 & ASN 1082 (2), TYR 1079 \\
\hline L & $\mathrm{CNC}(\mathrm{C})(\mathrm{C}) \mathrm{Cc} 1 \mathrm{nc}(\mathrm{CCCC} 2 \mathrm{ccccc} 2) \mathrm{no} 1$ & -6.2 & $\begin{array}{l}\text { CYS 1013, ALA } 1046 \text { (2), TYR 1079, ASN } \\
1082\end{array}$ \\
\hline M & $\begin{array}{l}\mathrm{C}=\mathrm{CCN}(\mathrm{CCN} 1 \mathrm{CCC}(\mathrm{C})(\mathrm{C}(=0) \mathrm{OC}) \mathrm{CC} 1) \mathrm{C}(\mathrm{C}) \operatorname{c1} \operatorname{ccc}(\mathrm{S}(=0) \\
(=0) \mathrm{N}(\mathrm{CC}) \mathrm{CC}) \operatorname{cc} 1\end{array}$ & -6.1 & $\begin{array}{l}\text { CYS 1013, ALA } 1046 \text { (2), TYR 1079, ASN } \\
\text { 1082, TRP } 1084 \text { (2), LEU 1205, MSE } 1242\end{array}$ \\
\hline $\mathrm{N}$ & $\mathrm{CC}(\mathrm{C}) \mathrm{N}(\mathrm{Cc} 1 \mathrm{ccccc} 1) \mathrm{Cc} 1 \mathrm{nnn} \operatorname{co} \mathrm{C}(\mathrm{C}) \mathrm{C}$ & -6.0 & CYS 1013, ALA 1046 (2), TYR 1079 (2) \\
\hline 0 & $\mathrm{CC}(\mathrm{C}) \mathrm{N}=\mathrm{C}(\mathrm{NCC} 1 \mathrm{cccs} 1) \mathrm{NCc} 1 \mathrm{ccco} 1$ & -6.0 & $\begin{array}{l}\text { ALA 1046, TYR 1079, TRP } 1084 \\
\text { (unfavorable) }\end{array}$ \\
\hline$P$ & $\operatorname{CCN}(\mathrm{C}) \operatorname{CCNC}(\mathrm{CCSC} 1 \operatorname{ccccc} 1) \mathrm{CN}(\mathrm{C}) \mathrm{CCc} 1 \mathrm{nn}[\mathrm{nH}] \mathrm{n} 1$ & -6.0 & $\begin{array}{l}\text { ALA 1046, TYR 1047, TYR 1079, ASN } 1082 \\
\text { TRP 1084, MSE 1242, ASP } 1246\end{array}$ \\
\hline Q & $\operatorname{COCCN} 1 \mathrm{C} 2 \mathrm{CCC} 1 \mathrm{CC}(\mathrm{N}(\mathrm{C}) \mathrm{CCCCN}(\mathrm{Cc} 1 \operatorname{ccccc} 1) \mathrm{Cc} 1 \operatorname{ccc} n 1) \mathrm{C} 2$ & -6.0 & TYR 1047 (2), TYR 1079, TRP 1084 \\
\hline
\end{tabular}

Table 2: Physicochemical properties, Leadlikeness and synthetic accessibility of the top 17 leads 


\begin{tabular}{|c|c|c|c|c|c|c|c|c|c|c|}
\hline Molecule & $\begin{array}{l}\text { MW } \\
(\mathrm{g} / \mathrm{mol})\end{array}$ & $\begin{array}{l}\text { Num. } \\
\text { rotatable } \\
\text { bonds }\end{array}$ & HA & HD & $\begin{array}{l}\text { TPSA } \\
\left(\AA^{2}\right)\end{array}$ & $\begin{array}{l}\text { Consensus } \\
\text { Log Po/w }\end{array}$ & $\begin{array}{l}\text { Water } \\
\text { solubility } \\
\text { class }\end{array}$ & $\begin{array}{l}\text { Bioavailability } \\
\text { score }\end{array}$ & Leadlikeness & $\begin{array}{l}\text { Synthetic } \\
\text { accessibility }\end{array}$ \\
\hline A & 316.44 & 2 & 4 & 3 & 48.56 & 1.94 & $\begin{array}{l}\text { Moderately } \\
\text { soluble }\end{array}$ & 0.55 & YES & 4.40 \\
\hline B & 283.37 & 5 & 4 & 2 & 54.77 & 1.46 & $\begin{array}{l}\text { Moderately } \\
\text { soluble }\end{array}$ & 0.55 & YES & 3.65 \\
\hline C & 407.60 & 13 & 4 & 2 & 123.69 & 3.39 & $\begin{array}{l}\text { Poorly } \\
\text { soluble }\end{array}$ & 0.55 & NO & 4.23 \\
\hline D & 302.44 & 6 & 3 & 1 & 81.54 & 2.59 & $\begin{array}{l}\text { Moderately } \\
\text { soluble }\end{array}$ & 0.55 & YES & 3.13 \\
\hline E & 377.53 & 10 & 5 & 1 & 69.53 & 1.05 & Soluble & 0.55 & NO & 3.81 \\
\hline $\mathrm{F}$ & 310.44 & 6 & 3 & 1 & 41.05 & 3.09 & $\begin{array}{l}\text { Poorly } \\
\text { soluble }\end{array}$ & 0.55 & YES & 3.44 \\
\hline G & 325.45 & 6 & 3 & 1 & 59.97 & 2.50 & $\begin{array}{l}\text { Moderately } \\
\text { soluble }\end{array}$ & 0.55 & YES & 3.96 \\
\hline $\mathrm{H}$ & 217.27 & 4 & 3 & 1 & 47.04 & 1.97 & $\begin{array}{l}\text { Moderately } \\
\text { soluble }\end{array}$ & 0.55 & NO & 2.60 \\
\hline I & 283.41 & 8 & 3 & 0 & 19.37 & 2.80 & $\begin{array}{l}\text { Poorly } \\
\text { soluble }\end{array}$ & 0.55 & NO & 2.21 \\
\hline J & 364.48 & 15 & 5 & 3 & 82.70 & 1.80 & $\begin{array}{l}\text { Moderately } \\
\text { soluble }\end{array}$ & 0.55 & NO & 3.58 \\
\hline K & 324.42 & 13 & 4 & 3 & 86.61 & 1.17 & $\begin{array}{l}\text { Moderately } \\
\text { soluble }\end{array}$ & 0.55 & NO & 2.89 \\
\hline L & 287.40 & 8 & 4 & 1 & 50.95 & 3.43 & $\begin{array}{l}\text { Poorly } \\
\text { soluble }\end{array}$ & 0.55 & NO & 3.36 \\
\hline$M$ & 479.68 & 13 & 7 & 0 & 78.54 & 3.50 & $\begin{array}{l}\text { Moderately } \\
\text { soluble }\end{array}$ & 0.55 & NO & 4.31 \\
\hline $\mathrm{N}$ & 272.39 & 6 & 3 & 0 & 33.95 & 2.58 & $\begin{array}{l}\text { Moderately } \\
\text { soluble }\end{array}$ & 0.55 & YES & 2.41 \\
\hline 0 & 291.41 & 8 & 2 & 2 & 77.80 & 2.87 & $\begin{array}{l}\text { Moderately } \\
\text { soluble }\end{array}$ & 0.55 & NO & 3.26 \\
\hline$P$ & 405.60 & 15 & 6 & 2 & 98.27 & 2.59 & $\begin{array}{l}\text { Poorly } \\
\text { soluble }\end{array}$ & 0.55 & NO & 4.12 \\
\hline Q & 450.66 & 13 & 5 & 0 & 31.84 & 3.91 & $\begin{array}{l}\text { Poorly } \\
\text { soluble }\end{array}$ & 0.55 & NO & 5.06 \\
\hline
\end{tabular}

\section{Figures}




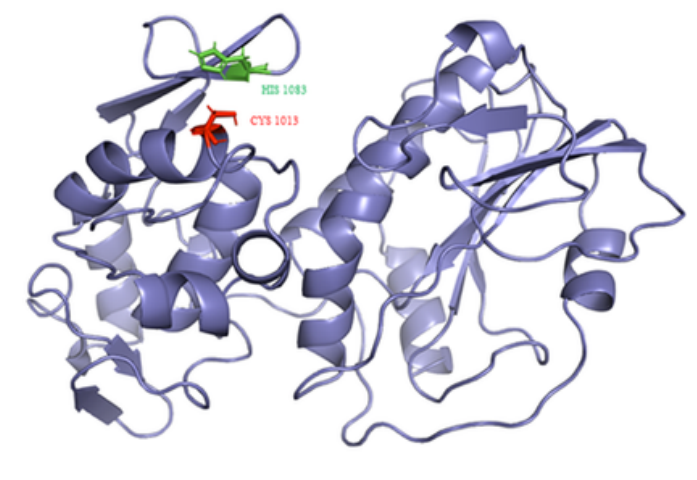

A

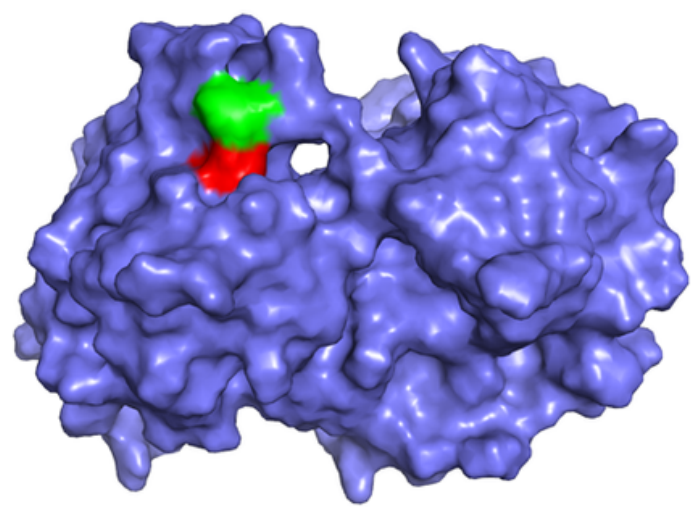

B

\section{Figure 1}

(A) Cartoon representation, (B) Surface representation of the CHIKV nsP2 protease (Red: CYS 1013, Green: HIS 1083) 

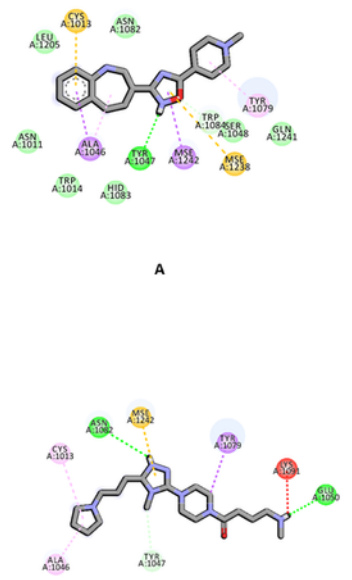

E

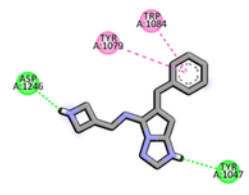

B

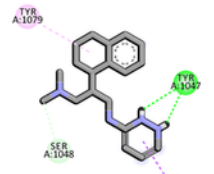

19.8.

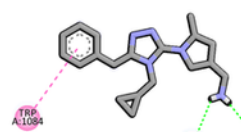

AS102

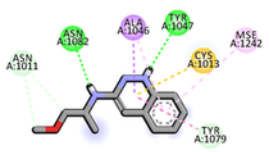

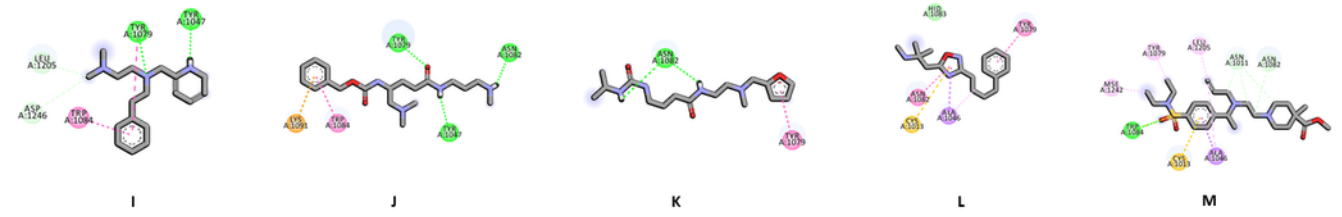

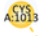

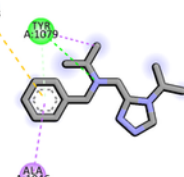

(1)

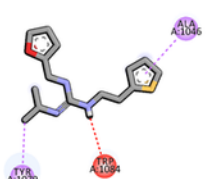

$\circ$

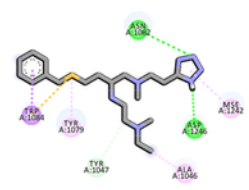

P

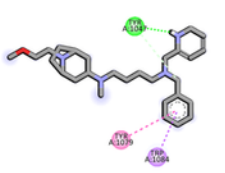

Q

\section{Figure 2}

2D depiction of protein-ligand interactions 